\title{
Frontline Experience against COVID-19 in a Tertiary Obstetrics and Gynaecology Hospital: A commentary
}

\author{
Chek Swee, Allison Tan ${ }^{1}$, Chye Lee Lily Kho ${ }^{1}$, Shu Qi Tan ${ }^{1}$, Rajeswari Kathirvel ${ }^{1}$, and \\ Suzanna Sulaiman ${ }^{1}$ \\ ${ }^{1}$ KK Women's and Children's Hospital
}

June 4, 2020

Frontline Experience against COVID-19 in a Tertiary Obstetrics and Gynaecology Hospital: A commentary Allison Tan CS ${ }^{1}$, Lily Kho $\mathrm{CL}^{1}$, Tan Shu $\mathrm{Qi}^{1}$, Rajeswari Kathirvel ${ }^{1,2}$, Suzanna Sulaiman ${ }^{1,2}$

${ }^{1}$ Department of Obstetrics and Gynaecology, KK Women's and Children's Hospital, Singapore

${ }^{2}$ Lee Kong Chian school of Medicine, Yong Loo Lin School of Medicine and Duke NUS, Singapore

Corresponding author: Dr Lily Kho CL

Email address: kho.chye.lee@singhealth.com.sg

Correspondence: Dr Lily Kho CL, Associate Consultant, Department of Obstetrics and Gynaecology, KK Women's and Children's Hospital, 100 Bukit Timah Road, Singapore 229899

Introduction

The COVID-19 outbreak is an ongoing pandemic that has taken the world by storm. As of $31^{\text {st }}$ May 2020 , there has been more than 6 million confirmed cases, including 371,000 deaths $^{[1]}$. Singapore was one of the earliest countries outside of China affected by COVID-19, with the first case diagnosed on $23^{\text {rd }}$ January 2020. By $31^{\text {st }}$ May 2020, the nation reported a total of 34,884 cases (the highest number in Southeast Asia) with 23 deaths ${ }^{[2]}$. In response to the outbreak, the Ministry of Health $(\mathrm{MOH})$ raised the Disease Outbreak Response System Condition (DORSCON) from yellow to orange on $7^{\text {th }}$ February $2020{ }^{[3]}$, adopting a heightened risk posture. This emphasized the urgency of containing the disease, inhibiting spread from person to person. The country further declared a 'Circuit Breaker', implementing a stringent set of social distancing measures from $7^{\text {th }}$ April 2020, extending to $1^{\text {st }}$ June 2020.

KK Women's and Children's Hospital (KKH) is Singapore's largest hospital exclusively treating children and women with obstetrics and gynaecology $(\mathrm{O} \& \mathrm{G})$ conditions. Its Urgent Obstetrics and Gynaecology Centre (UOGC) is a 24-hour walk-in centre for women with acute O\&G conditions. Approximately 2,700 patients access this service every month. The COVID-19 outbreak has posed several challenges to UOGC's daily operation, being one of the "gatekeepers" for the hospital. However, our centre was quick and dynamic in responding to the evolving outbreak in order to maintain continuity of care.

Logistical considerations

\section{Designation of an isolation area}

As one of the first measures implemented, we designated an isolation area that was physically separate from the main centre ${ }^{[4]}$. Three negative pressure consultation rooms have been assigned solely for the review of patients in isolation, alongside a segregated waiting area. Patients are seated one metre apart in the waiting 
area. One consultation room is equipped with an ultrasound machine to minimise cross contamination from the movement of patients in isolation to other departments for imaging. The consultation rooms are thoroughly cleaned and sanitised after each case.

\section{Manpower}

Hospital transmission has shown to pose a real and serious threat to healthcare personnel and patients ${ }^{[5]}$. To prevent cross contamination within UOGC and other areas of the hospital, a dedicated team of doctors and nurses is scheduled to service the isolation area. Each team consists of a senior and junior doctor, and two nurses working 12-hour shifts for 7 days in a row. Additional support for the isolation team is provided by a separate team consisting of Obstetricians, Gynaecologists and Infectious Disease (ID) physicians. This arrangement ensured that both the quality of care and safety of patients were not compromised during the crisis.

Standard precautions in operation prior to the COVID-19 outbreak included the practice of good hand hygiene. We adopted additional droplet and airborne precautions in the form of enhanced Personal Protection Equipment (PPE) in response to the outbreak ${ }^{[6]}$. This includes the use of N95 masks, gowns, gloves, goggles or visors and head caps. The Powered Air-Purifying Respirator (PAPR) is used for collection of nasopharyngeal swabs for COVID-19 testing or when performing any aerosol-inducing procedure. At the early stages of the outbreak, all hospital staff were fitted with appropriate size N95 masks and trained on the proper sequence of donning and removing PPE.

Apart from goggles and visors, the enhanced PPE is single use. A new set is donned for each patient. Used PPE items are discarded into yellow biohazard bags. The housekeepers in enhanced PPE then perform terminal cleaning on the consultation room after each use and promptly dispose of these biohazard bags. Equipment is cleaned and disinfected between use.

\section{Workflow considerations}

Another key aspect of UOGC's response to COVID-19 is its operational changes on the journey of patients in isolation (Figure A).

\section{Triage}

The journey of a patient in UOGC begins in the triage area. Patients were given a mask on entry into UOGC until it was made mandatory by the government for everyone to wear a mask outside their home. Each patient completes a declaration form used to stratify their risk. Patients with travel history as well as contact history with anyone suspected or confirmed to have COVID-19, fever, or acute respiratory symptoms are immediately isolated. Accompanying persons are submitted to the same screening criteria. The triage criteria is regularly updated based on the "suspect case" definition revisions by $\mathrm{MOH}^{[7]}$ in response to the evolving pandemic. During its first week of implementation, a positive travel history only included patients with recent travel to mainland China. This quickly expanded to include South Korea, then Japan, before finally including all persons with any travel history outside of Singapore when there was a significant rise in the COVID-19 cases globally.

\section{Consultation}

In addition to a standard obstetric and gynaecological history and examination, a detailed history of patients' respiratory symptoms, travel, and contact history are obtained. A dedicated team of ID physicians are readily available for phone consultation at all times. Patients deemed to be at low risk after review by the isolation doctor are transferred to general wards. The workflow has been regularly updated based on the evolving evidence on the condition.

We implemented protocols for isolated patients requiring formal imaging. When needed, portable chest radiographs were performed by radiographers in enhanced PPE within the isolation area. When these patients required formal ultrasound scans, they were transferred to the radiology department after alerting 
the relevant personnel in advance. The scan would then be performed by the radiologist in enhanced PPE in a dedicated isolation room.

We ensured that standard of care was not compromised for patients seen in isolation with modifications to our workflow and protocols in the UOGC. High risk treatment procedures such as nebulisation ${ }^{[8]}$ were administered in an alternative form with a metered-dose inhaler. Where this is not possible, nebulisation was administered in an isolation room in PAPR.

\section{Admission processes}

To minimise cross-contamination, we carefully planned the movement of isolated patients. In addition to a nurse and porter, patients for hospitalisation are escorted by two security officers in enhanced PPE to the designated isolation ward. The security officers play a vital role in ensuring that the patients in isolation do not come into close proximity with others by navigating the route. Lifts used are thoroughly disinfected immediately after.

\section{Discharge processes}

We initiated a Swab And Send Home (SASH) protocol for patients with fever or acute respiratory symptoms who did not have active gynaecological issues requiring admission. Prior to its implementation, all patients requiring COVID-19 swab testing were admitted. The SASH protocol eliminated the need for admission of these stable patients. Instead, these patients are issued a five-day hospitalisation leave, during which they have to remain isolated at home by law. They are given clear discharge advice defining the precautionary measures they should take as advised by the $\mathrm{MOH}$ and to return for further medical attention should their condition worsen. These swab results are communicated to the patients by the isolation team in a timely fashion.

A porter collects the discharge medications from the pharmacy on behalf of these patients to further minimise social contact.

\section{Inter-hospital transfer processes}

As our hospital is exclusively an O\&G hospital, women presenting with non-O\&G issues are transferred to other general hospitals. We modified the workflow for these women in isolation so that the transfers are made in a dedicated ambulance and the patients are offered direct admission in isolation areas of the receiving hospital, thereby reducing unnecessary contamination in the receiving hospital.

\section{Challenges encountered}

Setting up the isolation area was challenging. The department had to take into account the flow of patients through the centre, whilst ensuring segregation of other patients from those requiring isolation. The centre was able to achieve this with no impact on UOGC services and without having to create makeshift temporary clinics.

Response to an evolving outbreak is dynamic and the UOGC isolation team has to be quick to adapt to the latest recommendations by the hospital and $\mathrm{MOH}$. Figure B illustrates the timeline of changes implemented in UOGC. Our team works closely with the ID department, and regularly reviews and revises our protocols.

One of the difficulties encountered was false declarations made by patients at triage. This compromises the safety of our staff and other patients. Patients are forewarned of the consequences of false declaration, which may lead to prosecution under the Infectious Disease Act ${ }^{[9]}$ prior to signing it. Patients found to have breached Stay Home Notice or made false declarations were reported to the relevant government authorities.

The welfare of the isolation team is a key priority for our department. Stress and fatigue from working in the frontlines for extended shift hours are inevitable ${ }^{[10]}$. Dedicated en-suite call rooms are allocated to the isolation team to avoid cross contamination with the regular on-call team. Unwell staff are encouraged to seek medical attention and given medical leave. Doctors are granted dedicated rest following their week-long service. The hospital instituted a dedicated phone line for staff requiring mental and emotional support. 
Senior doctors took on shared workload to reduce manpower fatigue amongst the juniors. Regular feedback is gathered to optimise working conditions and streamline the workflow. The isolation team has received strong support from members of the public via letters of encouragement and gifts of gratitude.

\section{Conclusion}

Since the isolation team began operation, the UOGC has seen a total of 958 cases. As Singapore prepares for the next phases of 'Post Circuit breaker', the UOGC recognises the importance of continued vigilance. This crisis has challenged and tested the centre's ability to respond swiftly to an evolving pandemic. Continued reassessment and dynamic reviews of processes and workflows in place is the key to help us curb this outbreak and to prepare for the future.

\section{Acknowledgements}

The authors thank nurse clinician Riza Tungul; doctors, nurses, and support staff of the Urgent Obstetrics and Gynaecological Centre especially the dedicated "Isolation" teams; the Department of Obstetrics \& Gynaecology; Department of Infectious Diseases; Division of Obstetrics and Gynaecology, KK Women's and Children's Hospital, Singapore.

Disclosure of Interest

No conflict of interest from members of the study.

\section{Contribution to Authorship}

All authors contributed to study conceptualisation and design. ATCS wrote the original manuscript. The rest of the authors critically reviewed the content. All the authors have reviewed and approved the final version of the manuscript.

Details of Ethics Approval

No ethics approval.

Funding

No funding required.

\section{References}

[1] Covid19.who.int. 2020. WHO Coronavirus Disease (COVID-19) Dashboard. [online] Available at: $<$ https://covid19. who.int/?gclid=EAIaIQobChMIgafZrZzU6QIV1Z7CCh1xcA66EAAYASAAEgKFjvD_BwE $>$ [Accessed 31 May 2020].

[2] Moh.gov.sg. 2020. MOH | Updates On COVID-19 (Coronavirus Disease 2019) Local Situation. [online] Available at: <https://www.moh.gov.sg/covid-19> [Accessed 31 May 2020].

[3] Moh.gov.sg. 2020. MOH | News Highlights. [online] Available at: <https://www.moh.gov.sg/newshighlights/details/risk-assessment-raised-to-dorscon-orange> [Accessed 28 May 2020].

[4] Wilder-Smith, A. and Freedman, D., 2020. Isolation, quarantine, social distancing and community containment: pivotal role for old-style public health measures in the novel coronavirus (2019-nCoV) outbreak. Journal of Travel Medicine, 27(2).

[5] Wee, L., Conceicao, E., Sim, X., Aung, M., Tan, K., Wong, H., Wijaya, L., Tan, B., Ling, M. and Venkatachalam, I., 2020. Minimizing intra-hospital transmission of COVID-19: the role of social distancing. Journal of Hospital Infection, 105(2), pp.113-115.

[6] Who.int. 2020. Infection Prevention And Control During Health Care When Novel Coronavirus (Ncov) Infection Is Suspected. [online] Available at: <https://www.who.int/publications-detail/infection-preventionand-control-during-health-care-when-novel-coronavirus-(ncov)-infection-is-suspected-20200125> 
[7] Ministry of Health, 2020. Revision Of Suspect Case Definition For Coronavirus Disease 2019 (COVID 19) And Swab-And-Send-Home Criteria. Singapore. [Accessed 28 May 2020].

[8] Amirav, I. and Newhouse, M., 2020. Transmission of coronavirus by nebulizer: a serious, underappreciated risk. Canadian Medical Association Journal, 192(13), pp.E346-E346.

[9] Singapore Statutes Online. 2020. Infectious Disease Act 21A [online] Available at: https://sso.agc.gov.sg/Act/IDA1976?ProvIds=pr21A-\#pr21A- [Accessed 31 May 2020].

[10] Chua, M., Lee, J., Sulaiman, S. and Tan, H., 2020. From the frontline of COVID-19 - how prepared are we as obstetricians? A commentary. BJOG: An International Journal of Obstetrics \& Gynaecology, 127(7), pp.786-788

\section{Hosted file}

Figure A.docx available at https://authorea.com/users/329670/articles/456615-frontlineexperience-against-covid-19-in-a-tertiary-obstetrics-and-gynaecology-hospital-acommentary

\section{Hosted file}

Figure B.docx available at https://authorea.com/users/329670/articles/456615-frontlineexperience-against-covid-19-in-a-tertiary-obstetrics-and-gynaecology-hospital-acommentary 